\title{
A MODULAR STRUCTURAL DESIGN FOR \\ A SPECTROGRAPHICAL MMT
}

\author{
RubenS FREIRE FERRERO \\ Observatoire Astronomique \\ 67.000 - Strasbourg \\ F R A N C E
}

\author{
Michel CHEVRETON and Paut FELENBOK \\ Observatoire de Paris - Meudon \\ 92.190 - Meudon \\ F R A N C E
}

\section{Summary.-}

With the aim to propose alternative solutions for Multi Mirror Telescopes, we present some ideas about a MMT for High Resolution Spectroscopy purposes. The alternative solution is intended both in technical conception and in economical costs.

This possible project consider the use of a system of 36 mirrors about 1.5 meter in diameter, collecting light independently each other and transmitting the light down to the slit spectrograph by optical fibers.

To reduce mirror costs and structural weight, we propose to develop the "replique" technique over light-weighted supports modelled and adjusted over a master convex mirror.

Different project design for a modular hive structure are considered.

Proceedings of the IAU Colloquium No. 79: "Very Large Telescopes, their Instrumentation and Programs", Garching, April 9-12, 1984. 


\section{1.- Introduction.-}

At present time, there are many MMT projects in study at the USA and also in Europe:the ESO project The major encountered problem is the financial support of such a project, because of the new engineering conception, structural and optical design and new technical devices, but also because of construction and installation costs.

So, this kind of projects, could be only realized in an international cooperation as the ESO project or with the cooperation of several institutions as in the various USA projects.

Nevertheless, we can imagine a MMT project with a more restricted astronomical application (and consequently, with cheaper costs), that could be also undertaken in an european framework.

How could be realize a cheaper MMT with a high output and efficiency for the astronomical community?

We propose to study a MMT devoted only to spectrography (at high . resolution, and also, the capabilities for mean and low resolution) and neglecting imagery.

An ensemble of 1.50-meter telescopes will be linked to the spectrograph, by means of a system of optical fibers (Fig. 1 and 2). Light-weighted mirrors and special fabrication techniques, could reduce substantially fabrication, transportation and installation costs.

The support structures could be cheaper, supporting less weight and reducing the installation of sophisticated devices that concentrate all the light in only one optic way.

The description and characteristics of our MMT project are summarized in section 3 .

Now, our purpose is to begin the developpement of such a project, having in mind that our idea is complementary of the other MMT projects in process, and also, that independant reflections about different alternative projects, may finally profit all concerned astronomers, favouring a more fruitful discussion allowing to find the best solutions for our urgent astronomical needs. 


\section{2.- Astronomical goals.-}

What kind of astronomical studies could be developped with an Spectrographic MMT ?

The striking scientific goals that would be attained are principally those concerning High Resolution Spectroscopy (HRS) :

(a) for bright objects, reducing exposure time, rising the $S / N$ ratio (over 500 ) and spectral resolution (better than $100 \mathrm{~mA}$ ) allowing the discovery of fainter spectral features or rapid variabilities just now smeared on noise or not observed because the use of a limited spectral resolution ( instrumental profile);

(b) for faint objects $\left(11<m_{v}<15\right)$, giving the possibility to observe their spectra at HRS or to detect variabilities in line profiles.

Other worthwhile studies would be those concerning :

(c) for faint objects, and moderately spectral resolution ( MSR), the anaTysis of spectra and continua;

(d) for very faint objects $\left(15<m_{v}<17\right)$, and low spectral resolution ( LSR ), the study of spectra and continua and also, spectrophotometric analysis.

Some of the possible subjects that could be developped with such spectrographic MMT are summarized in the following Table I.

3.- Description and general characteristics of the Spectrographical MMT.-

The Spectrographical MMT would be composed by 36 mirrors of 1.5 meter in diameter, with a $5.25 \mathrm{~m}$ of focal distance, disposed in three concentrical rings around the 1.5-meter guide mirror, forming a cellular structure like a hive ( Fig. 1 and 2). In Table II, we summarize the optical characteristics of this MMT.

The total surface of active mirrors would be of about $63.5 \mathrm{~m}^{2}$, with an effective area of about $60 \mathrm{~m}^{2}$, equivalent to an individual mirror of approximately 9-meter diameter.

For the moment we retain the prime focus configuration (Fig. 2), considering that we could dispose of 1.5-meter parabolic mirrors. This configuration allow to reduce luminosity losses normally produced over an optical system (beam-recombined mirrors, prismes) that send the luminous beam to the spectrograph. 


\begin{tabular}{|c|} 
TABLE \\
ASTROPHYSICAL I \\
\hline
\end{tabular}
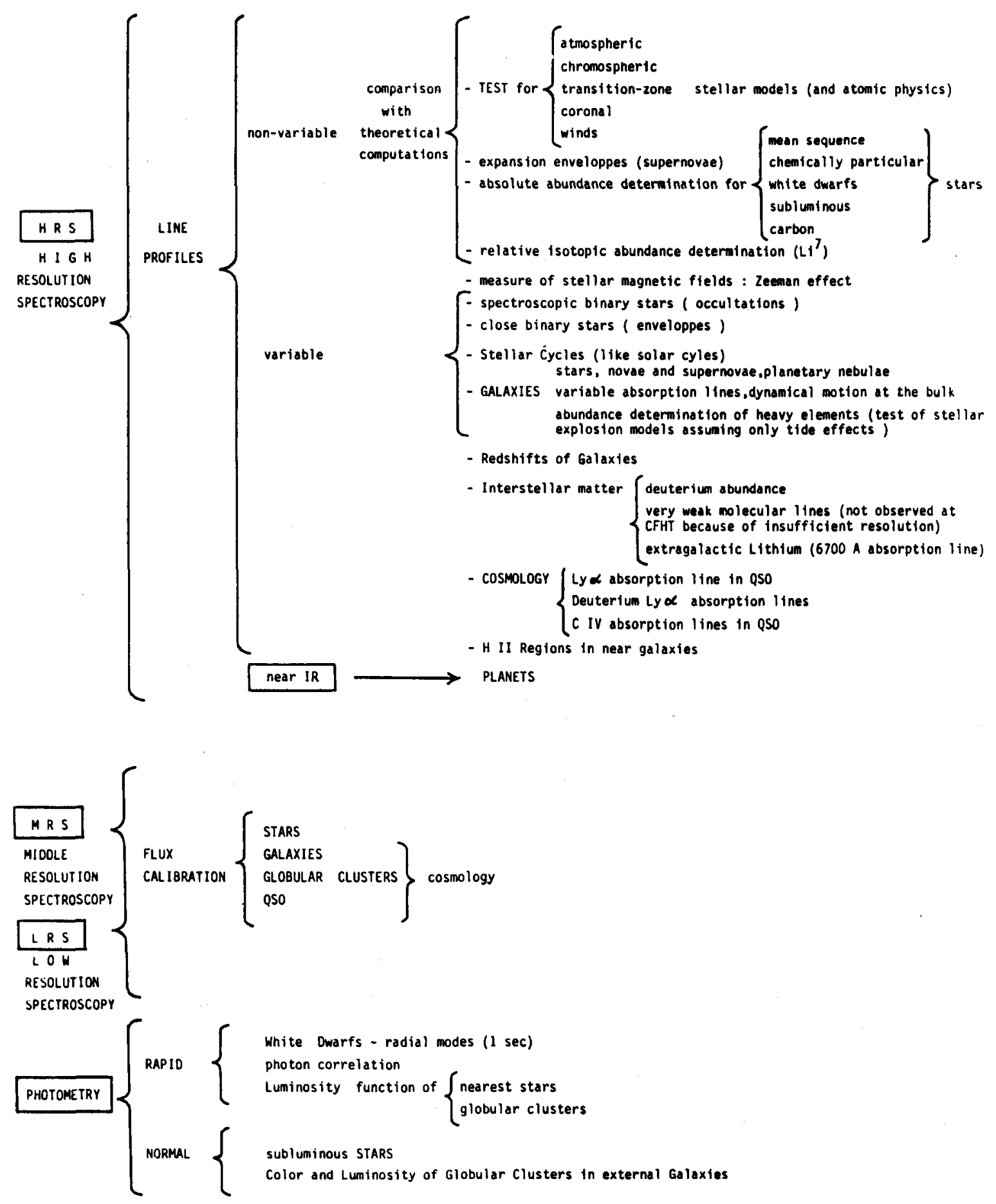

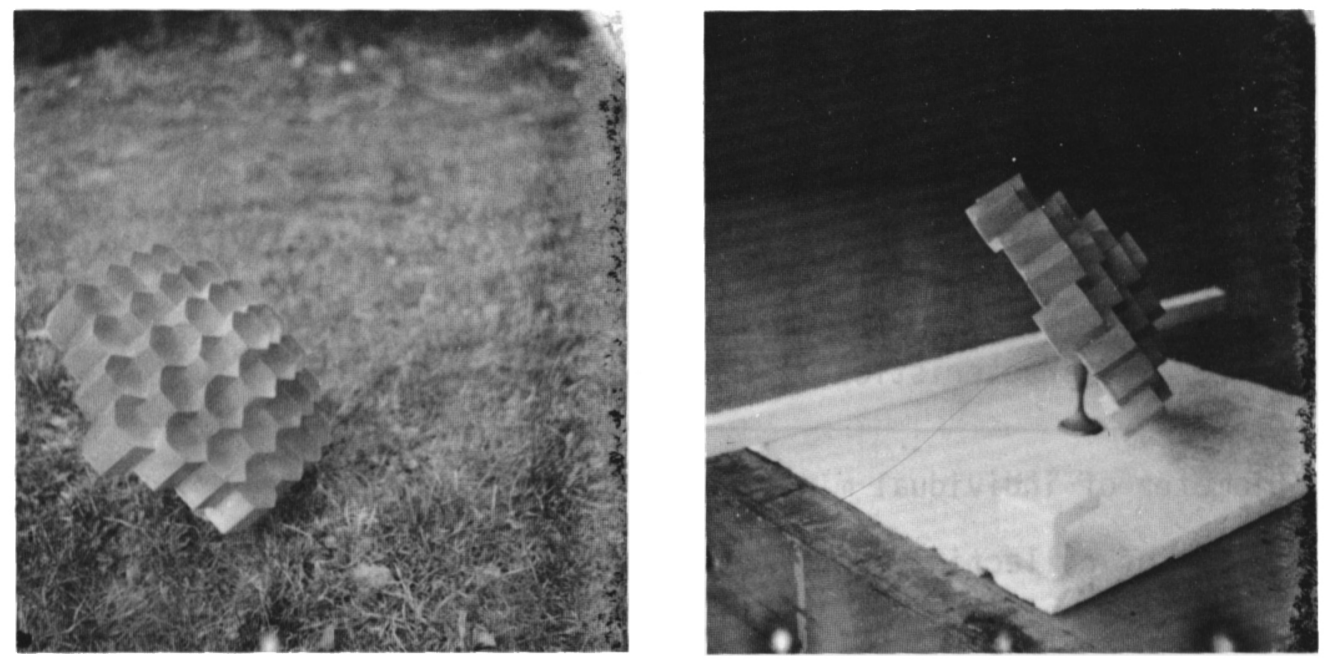

Fig. $1 a, b$

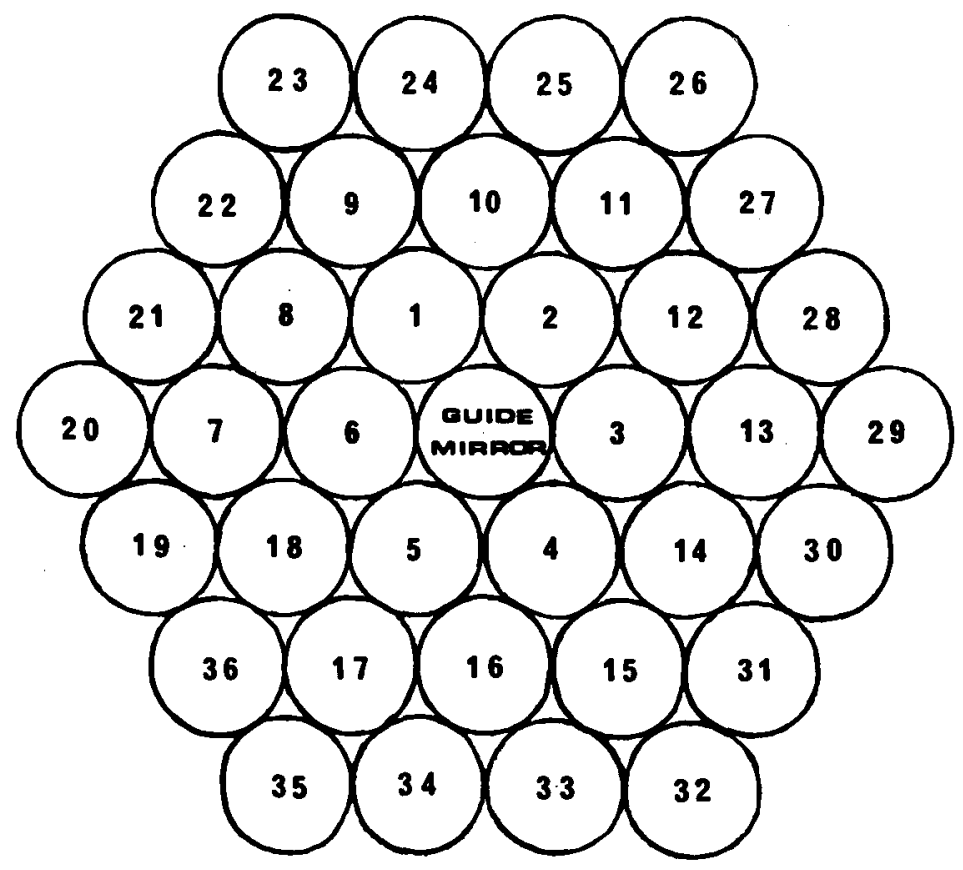

Fig. 2 
TA B LE I I

General characteristics of the Spectrographical MMT

\begin{tabular}{|c|c|}
\hline Diameter of individual mirrors & $1.5 \mathrm{~m}$ \\
\hline Number of collecting mirrors & 36 \\
\hline Total collecting area & $60 \mathrm{~m}^{2}$ \\
\hline One-mirror equivalent diameter & $9 \mathrm{~m}$ \\
\hline Aperture of individual mirrors (prime focus) & $f / 3.5$ \\
\hline Focal distance & $5.25 \mathrm{~m}$ \\
\hline Central-core diameter of the silicon optical fibers & $50 \mu$ or $2^{\prime \prime}$ \\
\hline External diameter of the optical fibers & $125 \mu$ \\
\hline Height of the entrance slit of the spectrometer & $4.5 \mathrm{~mm}$ \\
\hline \multirow[t]{2}{*}{ Aperture of the spectrograph : } & $f / 3$ \\
\hline & $f / 3$ \\
\hline Equivalent exit-slit of the spectrograph & $50 \mu \times 4500 \mu$ \\
\hline Detector : C C D (TI, Texas Instruments) & $800 \times 800$ pixels \\
\hline Pixel area & $15 \mu \times 15 \mu$ \\
\hline
\end{tabular}




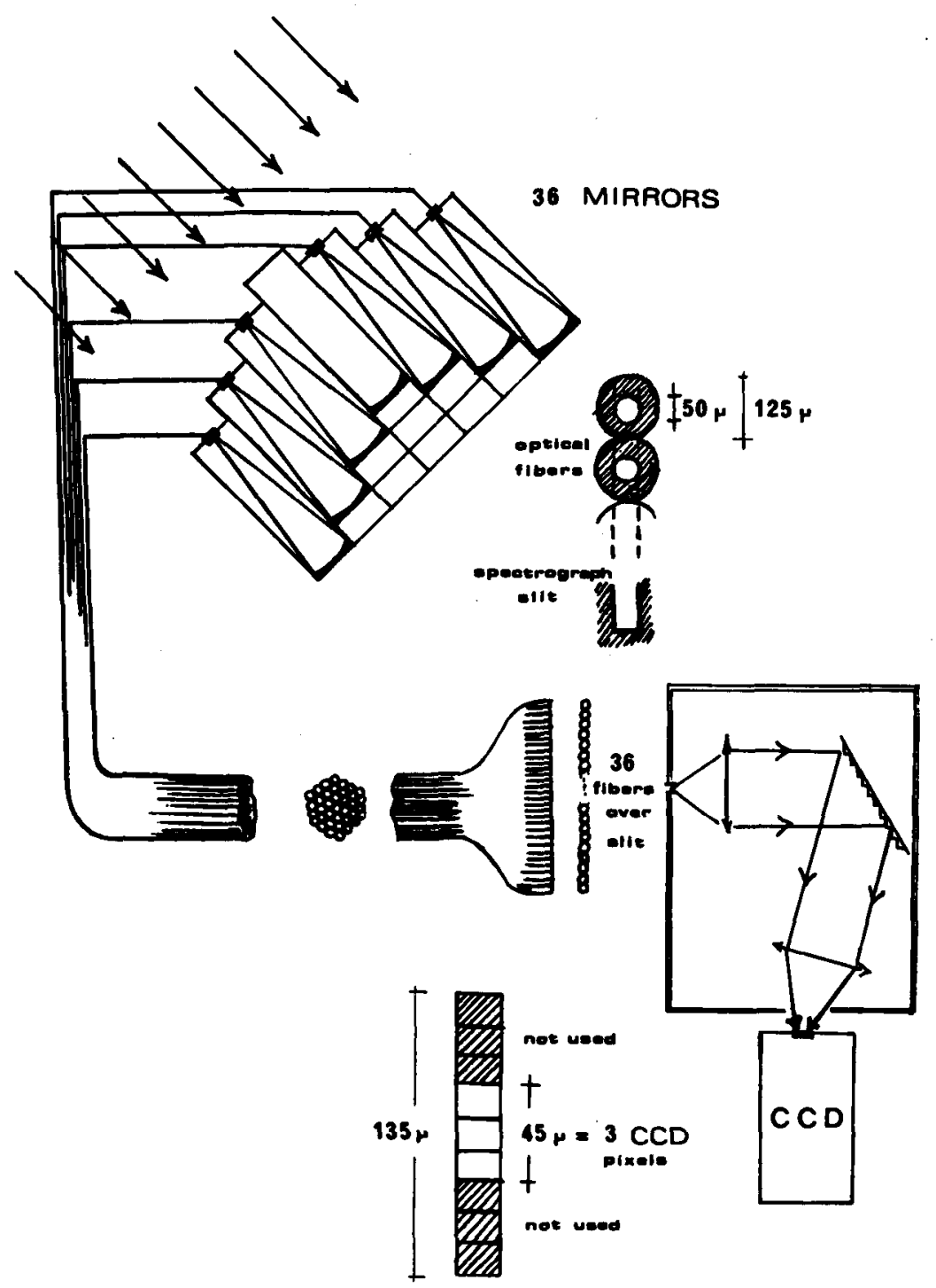

Fig. $\quad 3$ 
A system of optical fibers of $50 \mu$ diameter (corresponding to 2" field), with and end at the prime focus of each mirror, collect the light over the seeing image, and transmit it down independently: a 11 optical fibers are assembled at the other end over the spectrograph slit, covering a $50 \mu \times 4.5 \mathrm{~mm}$ slit entrance area (Fig. 3).

The spectrograph is conceived to give an exit aperture of $f / 3$ for an entry aperture of $\mathrm{f} / 3$. In this way, the effective exit slit would have a $50 \mu \times 4.5 \mathrm{~mm}$ area that could be analyzed by 3 CCD pixels of $15 \mu \times 15 \mu$. 4.- Structural design.-

The retained structural design (Fig. 1 and 2), provide us the possibility to study the changes of mechanical resistance for one ring to another, and offer in principle advantages also in the mounting of the structure: either module could be constructed in a standard way and assembled at the support structure independently of others.

At present, two alternative solutions would be considerated: an alt-azimuthal configuration and an equatorial configuration, both of them supported by a central column structure (Freire Ferrero, 1984).

This preliminary design intends to ga in in compactness: the basic idea is that the center of gravity of the support structure of mirrors, could be displaced in a little volume when the whole structure turns.

Schematic drawings of these two solutions are shown in Fig. 4 and 5.

The equatorial configuration could be considered assuming a bearing ball that support the structure of the ensemble of mirrors. Major problems must be presented to communicate equatorial motion to the hive structure. This kind of solution could be also used in an alt-azimuthal configuration.

Rough structural analysis show that maximum deformations of the mirror-support structure, could be reduced to the order of atmospheric seeing.

To move the mirrors, we can also consider la possibility to place a very fast motion's device for pointing the telescope in the case of the one column solution. This device could be formed by an array of wire cable drived by winchs that move around the whole structure,over a circular rail. 

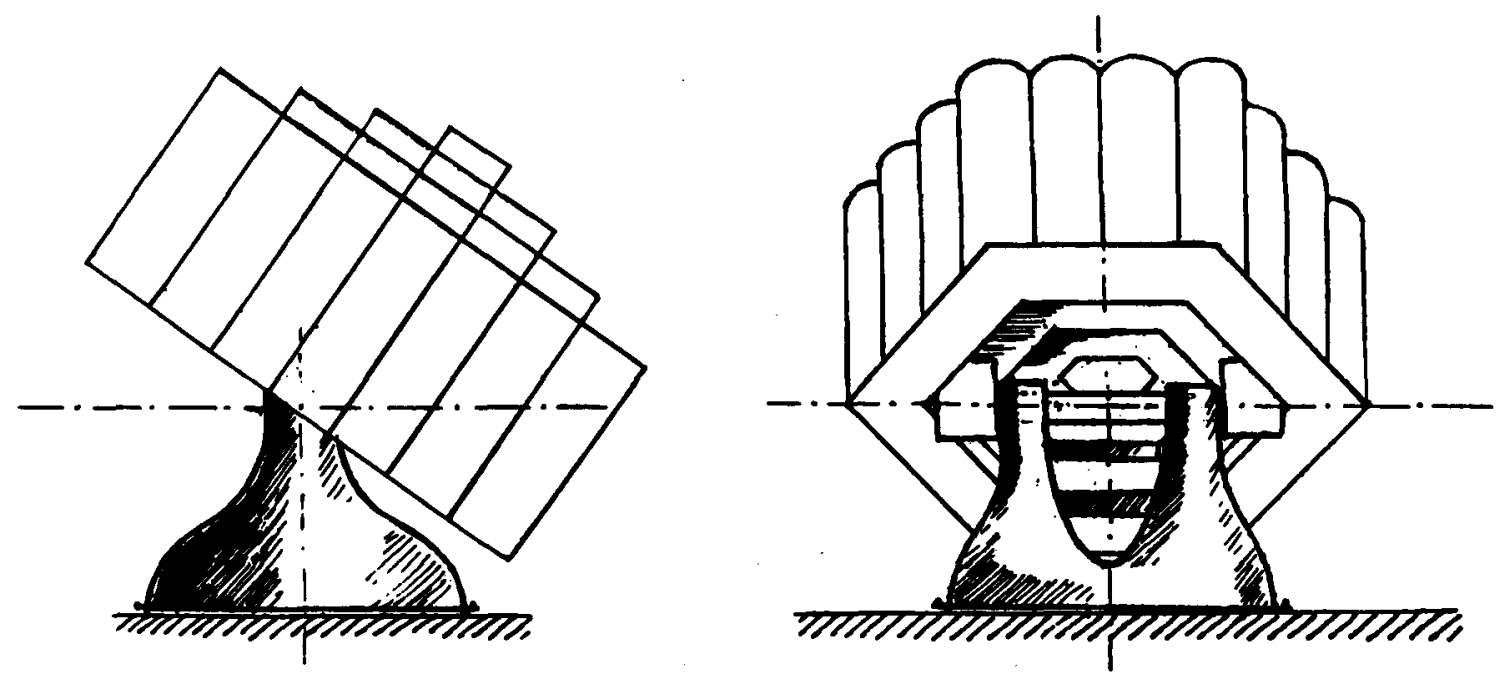

Fig. 4
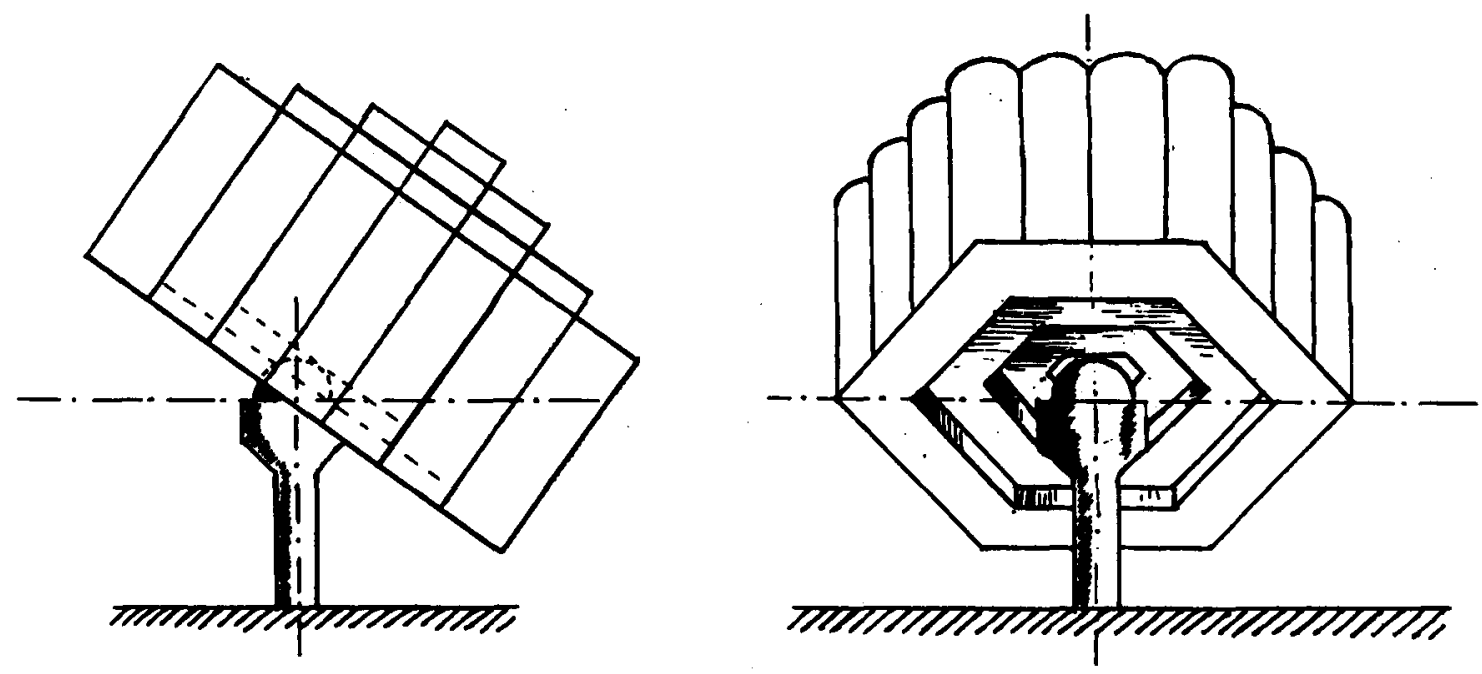

Fig. $\quad 5$ 
References.-

Angel J,Adams M.,Boroson T., Moore R.,1977, A very large optical telescope array linked with fused silica fibers, Astrophys $\mathrm{J} 218,776$.

Cayrel R., 1981, Les problèmes d'astrophysique dont l'étude nécessite une haute résolution spectrale, in 2è colloque du Télescope Spatial, Orsay, France.

Chevreton M. ,Cuisinier M., Felenbok P.,1981, Pré-étude du Projet "GRAFOS", grand recepteur astronomique a fibres optiques pour spectrométrie, rapport interne,Observatoire de Meudon.

Freire Ferrero R. Pré-étude de la structure de support d'un Très Grand Collecteur par la méthode des éléments finis, Thèse d'Ingénieur CNAM, Paris, in progress.

Hubbard E.,Angel J.,Gresham M., 1979, Operation of a long fused silica fiber as a link between telescope and spectrograph, Astrophys $\mathrm{J} 229,1074$.

Lequeux J.,1983, in Proc. Workshop ESO's Very Large Telescope, Cargèse 16-19 May 1983,ed.J.P. Swings and K. Kjär, ESO,Garching.

Osterbrok D.E., 1979, in The MMT and the future of ground based astronomy, ed. Weekes T.C., SAO Sp.Rep $N^{\circ} 385, \mathrm{p} 139$.

Weekes T.C. (ed.), 1979, The MMT and the future of ground based astronomy, Symp. May 9,1979, SAO Sp Rep $N^{\circ} 385$, SAO, Cambridge, Mass. 